\title{
One-dimensional chaos in a system with dry friction: analytical approach.
}

\author{
Nikita Begun $^{a}$, Sergey Kryzhevich ${ }^{a, b, \dagger}$ \\ ${ }^{a}$ Faculty of Mathematics and Mechanics, Saint-Petersburg State University, \\ 28, Universitetskiy pr., Peterhof, Saint-Petersburg, 198503, Russia; \\ ${ }^{b}$ University of Aveiro, Department of Mathematics, 3810-193, Aveiro, Portugal; \\ ${ }^{c}$ BCAM - Basque Center for Applied Mathematics, Mazarredo, 14, E48009 Bilbao, Basque- \\ Country, Spain.
}

\begin{abstract}
A simple mechanical system consisting of a mass and a dry friction element is considered. The mathematical model of such mechanical system is described. We show that the considered system corresponds to a skew product over a piecewise smooth mapping of a segment. We demonstrate that this base map for some value of parameters a robust chaotic dynamics can be observed.
\end{abstract}

Keywords: Li-Yorke chaos, mappings of segments, dry friction, reduction of dimension.

\section{Introduction.}

Systems with dry friction form a wide class inside discontinuous dynamical systems. They appear in many applications, especially in manufacturing systems: vibrating conveyors, percussion drilling, metal cutting, etc (see $2,3,4,5,6,12,13,14,16,19,22,23$, and references therein for review). Their properties manifest many principle differences with ones of smooth dynamics. For instance, the uniqueness theorem is not valid any more. An approach to study such systems has been developed by A.F. Filippov [7. He offered to consider piecewise continuous systems of differential equations as families of vector fields, defined on disjoint domains of the phase space and define auxiliary tangent flows on boundaries, respecting limit directions of vector fields. This approach reduces a discontinuous system to differential inclusions. Moreover, the phase space may become multidimensional i.e. a set of initial data of the full dimension may be transferred to a set of a lower dimension. The theory of discontinuous systems and specific bifurcation is well-developed [2,3,5,7,10,14,16,18,22, It

$\overline{1}$ Corresponding author.

Email address: kryzhevicz@gmail.com 
is also well-known that chaotic dynamics frequently occurs in such systems, particularly in ones with dry friction $[1,2,3,4,6,8,16,17,19,20,22,23]$.

Apart from numerical and experimental simulations, the most common analytic approach involves a reduction of dimension. For some systems with a dry friction it is possible to demonstrate that there exists an invariant set of dimension 1 where the attractor resides. A method to find this attractor has been developed by M. Wiercigroch, E. Pavlovskaya and A.Krivtsov in papers [12 13] and, in its general form, in the paper [17]. In our paper we use some ideas of this approach. Another powerful method has been proposed by R. Szalai and H. M. Osinga [20]. They have proved that for a general class of systems with a dry friction that the attractor resides in a polygon type set and demonstrated a possibility of a chaotic dynamics there. Later [21] they have shown, using a modification of their method that some complex structures like Arnold tongues can be observed in a neighborhood of the so-called grazing-sliding bifurcation [2,3,18].

The main aim of this paper is to provide a new method which allows to find chaotic invariant sets in systems with a dry friction. To demonstrate this method, we use a very simple example of a system with dry friction, first considered be A. Krivtsov and M. Wiercigroch [12]. First of all, we show that the considered dynamical system engenders a discontinuous mapping of a segment. Here we use ideas from [17. Then we study properties of this mapping which allows us to find two disjoint segments such that the image of every one of them covers their union. Moreover, we prove that the considered mapping is continuous on the union of these segments. This allows us to apply well-known techniques of one dimensional dynamics [11, Part 3, Section 15] and to demonstrate that a kind of chaotic dynamics, similar to one described by T. Li and J. Yorke [15] is there.

The main advantages of the offered method are the following.

(1) We can obtain chaotic sets which, in general, are not attractors.

(2) For simple systems with dry fiction, the offered method gives coefficient type criteria of chaos.

(3) Though we need a presence of a small parameter in our proofs, it is possible to estimate numerically how small this parameter must be. The presence of chaotic invariant sets does not correspond to a neighborhood of any bifurcation.

(4) The presence of chaotic behavior (but not corresponding invariant sets) is robust.

(5) A corresponding invariant measure can be described using techniques of [11, Part 3, Section 15].

The paper is organized as follows. In Section 1 we introduce the mathematical model of the considered system and describe possible regimes of motion. In Section 2 we define the main object of our investigation: the 1 dimensional mapping, corresponding to phases of switching for solutions. For this, we describe all possible scenarios of behavior of solutions. In the next section we describe the main properties of the introduced mapping. The main 
part of the proof is given in Section 4 where we study how the segments of continuity of the constructed mapping look like and find out two segments of continuity whose images cover their union. Section 5 is technical. We would like to prove existence of periodic points of all possible periods but we cannot apply the theory of mappings of a segment directly since we deal with a discontinuous mapping. However, still we can use standard methods of this theory to finish our proof. The main result is formulated in Section 5. A discussion, including notes on the robustness of the obtained set and some plans on the future research is given at Section 7 .

\section{Description of the mathematical model.}

Consider a single degree-of-freedom mechanical system, consisting of a point mass and a delimiter with dry friction (Fig. 1) which gives a simple model of percussion drilling. This system consists of a unit mass, whose motion is controlled by a harmonic external force $F(t)$ which is a sum of a positive constant component equal to $2 b$ and a harmonic component of a positive amplitude $a$ and a period equal to $2 \pi$. Also, the considered system includes a delimiter which provides an additional dry friction as soon as the mass reaches it. The maximal value of this friction is $q$. Here and later we always suppose that all considered parameters are non-dimensional.

Our main aim is to prove that provided some additional conditions are satisfied, the dynamics of the considered system is robustly chaotic in topological sense. Let $x$ be the current position of the mass and $y$ be one of the delimiter. We assume that the inequality $x \leq y$ is always satisfied i.e. the mass cannot penetrate through the delimiter and that the delimiter cannot move to the left so the value $y$ is always non-decreasing.

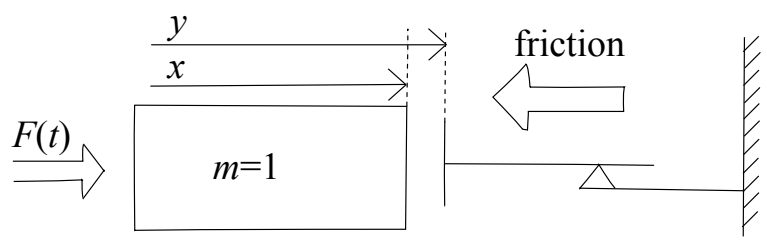

Fig. 1. The considered mechanical system.

Consider the value $\vartheta_{0} \in[0, \pi / 2]$ such that

$$
\pi-\vartheta_{0}=\cot \left(\vartheta_{0} / 2\right)
$$

This value is unique and $\vartheta_{0} \approx 0.81047, \sin \vartheta_{0} \approx 0.724611$.

We make the following assumptions on the parameters of the system:

$$
a>0, \quad b>0, \quad q \in\left(a \sin \vartheta_{0}, a\right) .
$$


We shall always suppose that $b \ll a$ (which implies $b \ll q$ ). This means that we always suppose that the ratio $b / a$ is as small as necessary.

There are five types of motions of the considered system.

(1) Free motion (f). This motion takes place if $x<y$ i.e. the mass and the delimiter do not interact. Then

$$
\ddot{x}=F(t)=a \sin t+2 b ; \quad \dot{y}=0 .
$$

(2) Progression (p). In this regime we have an additional friction. The motion is defined by equations

$$
\ddot{x}=F(t)-q=a \sin t+2 c=a \sin t+2 b-q ; \quad y=x .
$$

(3) Stop (s). Here the mass and the delimiter are both immobile, i.e.

$$
x=y, \quad \dot{x}=\dot{y}=0 .
$$

(4) Instantaneous stop (is). This happens if Condition (5) is satisfied for a fixed instant of time but is not true in its vicinity. So, this happens if the system switches from or to free motion or from/to motion with progression.

(5) Transition from the free regime to motion with progression (fp). This is an instant such that the mass was moving in a free regime before and switches to motion with progression there.

We always suppose that the free motion, motion with progression and the stop regime are observed at open intervals of time. This allows us to classify all instants of transition.

Let $t_{0}<t_{1}$ be zeros of the function $F(t)-q$ and $t_{2}<t_{3}$ be ones of the function $F(t)$ on $[0,2 \pi]$. Later on we consider the phase $\varphi=t \bmod 2 \pi$. Here $\varphi \in S^{1}=\mathbb{R} / 2 \pi \mathbb{Z}$.

Now we describe how we may proceed from one regime to another.

If the mass, moving according to Eq. (3) collides with the delimiter with a non-zero velocity, it proceeds to the progression regime. The transition $(\mathbf{f}) \rightarrow($ is $) \rightarrow(\mathbf{s})$ is possible at $t=\theta_{0}$ if and only if $x\left(\theta_{0}\right)=y\left(\theta_{0}\right), \dot{x}\left(\theta_{0}\right)=0$. If $F\left(\theta_{0}\right)>0$ then $\dot{x}(t)$ is negative before $t=\theta_{0}$ which is impossible. If $F\left(\theta_{0}\right)<0$, the mass returns back, "ignoring" presence of the delimiter. We have an instantaneous stop there. Otherwise, $F\left(\theta_{0}\right)=0$. If $\dot{F}\left(\theta_{0}\right)<0$ then again we have $\dot{x}(t)<0$ in a left neighborhood of $\theta_{0}$. So, $\dot{F}\left(\theta_{0}\right) \geq 0$ and, consequently, $\theta_{0}=t_{3} \bmod 2 \pi$. Then we have an instantaneous stop and the stop regime later on.

The progression regime is characterized by condition $\dot{x}>0$. As soon as the solution $x(t)$ reaches its maximal value, corresponding to $t=\theta^{\prime}$, the mass stops and then proceeds to free motion if $F(t)<0$ in a right neighborhood of $\theta^{\prime}$. Also, we may proceed to the "long" 
(non-instantaneous) stop regime if $F(t) \in[0, q]$ there. Otherwise, we stay in progression regime. Anyway an instantaneous stop takes place.

The system stays in stop regime until we have $F(t)<0$ or $F(t)>q$. In the first case we proceed to the free regime, in the second one we start progression. See Fig.2 for illustration.

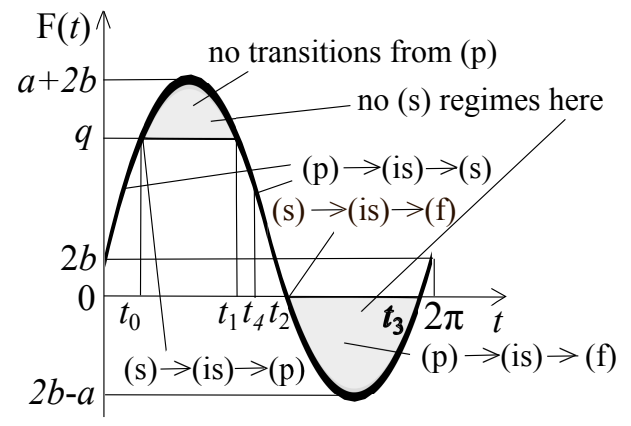

Fig. 2. Possible regimes of the system and transitions according to the phase.

\section{Reduction to dimension 1.}

Solutions of Eqs. (3)), (4) and (5) can easily be written down. If $x\left(\theta_{0}\right)=x_{0}, \dot{x}\left(\theta_{0}\right)=x_{1}$ we have

$$
x(t)=-a \sin t+b\left(t-\theta_{0}\right)^{2}+\left(x_{1}+a \cos \theta_{0}\right)\left(t-\theta_{0}\right)+x_{0}+a \sin \theta_{0}
$$

for Eq. (3) (free motion) and

$$
x(t)=-a \sin t+c\left(t-\theta_{0}\right)^{2}+\left(x_{1}+a \cos \theta_{0}\right)\left(t-\theta_{0}\right)+x_{0}+a \sin \theta_{0}
$$

for progression. For stop regime we always have $x_{1}=0$ and $x(t) \equiv x_{0}$.

Let us show that none of the considered regimes can be eternal.

Starting from the free motion, the mass will always return to the delimiter since $b>0$. Then we immediately proceed to progression motion unless we reach the delimiter with a velocity equal to zero. Let this zero velocity approach happens at the instant $t$ which corresponds to the phase $\tau$. Note that $\tau \in\left(t_{2}, t_{3}\right]$ otherwise the velocity would be negative in a left vicinity of $\tau$. Then if $\tau \in\left(t_{2}, t_{3}\right)$ the mass stops and starts moving in the free regime immediately after that. If $\tau=t_{3}$ the mass stops until the next instant $t_{0}+2 \pi k$.

In the motion with progression the derivative $\dot{x}$ vanishes soon or later since $c<0$. If this happens when $\varphi \in\left[t_{2}, t_{3}\right)$ we immediately proceed to free motion. Otherwise, the mass stops. If this happens for $\varphi \in\left[t_{1}, t_{2}\right)$ the mass stops until $\varphi=t_{2}$ and then switches to free motion. Note, that progression cannot be stopped while $t \in\left[t_{0}, t_{1}\right)$. If it is stopped on $\left[t_{3}, t_{0}\right)$ the mass waits the next instant $t_{0}+2 \pi k$ and then starts moving according to Eq. (44). In 
this case, we have $\theta_{0}=t_{0}$ and $x_{1}=0$ in Eq. (7). Consequently,

$$
\dot{x}(t)=-a\left(\cos t-\cos t_{0}\right)+2 c\left(t-t_{0}\right) .
$$

This function increases (and, therefore, cannot vanish) until $t=t_{1}$. However, since $b \ll q$, we may say that $\cos t_{3}>\cos t_{0}$ and the right hand side of Eq. (8) is negative for $t=t_{3}$. So, the motion stops somewhere at $\left[t_{1}, t_{3}\right)$ and then proceeds to the free flight regime. The stop regime may be finished by a transition to a free motion at $t=t_{2}+2 \pi k$ or by transition to the motion with a progression at $t=t_{0}+2 \pi k$. It is impossible neither on $\left(t_{0}, t_{1}\right)$ nor on $\left(t_{2}, t_{3}\right)$.

All together, this means that wherever and whenever the motion starts, finally, it has a transition to a free motion via an instantaneous stop.

Take an initial instant $\theta$ of such transition. There we have $\theta \in\left[t_{2}, t_{3}\right], x(\theta)=y(\theta)$ (without loss of generality, we may assume that this value is zero) and $\dot{x}(\theta)=0$. Then the value $\theta$ uniquely defines the farther dynamics.

If $T(\theta)$ is the phase of the next transition to free motion from stop or progression, it is uniquely defined by $\theta$. So, we may consider the $1 \mathrm{D}$ mapping $T:\left[t_{2}, t_{3}\right) \circlearrowleft$ which is, generally speaking, discontinuous. Considering this mapping, we lose some information about initial dynamical system, for instance, we do not know any more how the delimiter is shifted.

One of the following scenarios takes place for the considered motion.

(1) Scenario A: $(\mathbf{f}) \rightarrow(\mathbf{f p}) \rightarrow(\mathbf{p}) \rightarrow($ is $) \rightarrow(\mathbf{f})$;

(2) Scenario B: (f) $\rightarrow$ (fp) $\rightarrow$ (p) $\rightarrow$ (is) $\rightarrow$ (s) $\rightarrow$ (is $) \rightarrow(\mathbf{f})$;

(3) Scenario C:

$$
(\mathbf{f}) \rightarrow(\mathbf{f p}) \rightarrow(\mathbf{p}) \rightarrow(\text { is }) \rightarrow(\mathbf{s}) \rightarrow(\text { is }) \rightarrow(\mathbf{p}) \rightarrow(\text { is }) \rightarrow(\mathbf{s}) \rightarrow(\text { is }) \rightarrow(\mathbf{f})
$$

(4) Scenario D:

$$
(\mathbf{f}) \rightarrow(\mathbf{f p}) \rightarrow(\mathbf{p}) \rightarrow(\text { is }) \rightarrow(\mathbf{s}) \rightarrow(\text { is }) \rightarrow(\mathbf{p}) \rightarrow(\text { is }) \rightarrow(\mathbf{f})
$$

Also, there are two degenerate scenarios, corresponding to a zero-velocity stop of the free motion corresponding to $t=t_{3}+2 \pi k$ :

(1) Scenario C':

$$
(\mathbf{f}) \rightarrow(\text { is }) \rightarrow(\mathbf{s}) \rightarrow(\text { is }) \rightarrow(\mathbf{p}) \rightarrow(\text { is }) \rightarrow(\mathbf{s}) \rightarrow(\text { is }) \rightarrow(\mathbf{f})
$$

(2) Scenario D':

$$
(\mathbf{f}) \rightarrow(\text { is }) \rightarrow(\text { s }) \rightarrow(\text { is }) \rightarrow(\mathbf{p}) \rightarrow(\text { is }) \rightarrow(\mathbf{f})
$$


Here we omit all possible instantaneous stops after which the motion returns to the same regime. This does not hurt to equations of motion. However, such stops play an important role since they correspond to discontinuities of stroboscopic mappings. Let us study them more carefully.

\section{Properties of the mapping $T$.}

Let us introduce two auxiliary mappings $T_{1}$ and $T_{2}$. The first one corresponds to the first after $\theta$ approach to the delimiter. Denote the corresponding instant by $\theta_{1}$, i.e. $T_{1}(\theta)=\theta_{1}$. Note that the image of $T_{1}$ is an instant, not phase, so it can be greater than $2 \pi$.

The value $\theta_{1}$ corresponds to the first zero of the equation

$$
b(t-\theta)^{2} / a=\sin t-\cos \theta(t-\theta)-\sin \theta .
$$

satisfying the condition $t>\theta$.

The left hand side of Eq. (9) is always positive and proportional to the small parameter $b / a$. The right hand side is initially positive and grows faster than the left hand side. This is the distance between the graph of sine function and the tangent line to it, drawn at $\theta$. Note that $\sin \theta \leq-b / a<0$. If $\theta \in(3 \pi / 2,2 \pi)$ i.e. $\cos \theta>0$, the graph and its tangent line intersect once again on $(\theta,+\infty)$ and, therefore $\theta_{1}-\theta<2 \pi$. Otherwise, they do not intersect and, for small $b$, there exists a constant $C>0$ which does not depend on $\theta$ and is such that

$$
\theta_{1}-\theta \geq \frac{C a}{b} \cos \theta
$$

The mapping $T_{1}$ is, in general, discontinuous. All possible discontinuities correspond to the case when $\theta_{1}$ is not a simple zero of (9). Namely, derivatives of the left and the right hand side of (9) coincide for $t=\theta_{1}$ which means that the following condition is satisfied

$$
-a \cos \theta_{1}+2 b\left(\theta_{1}-\theta\right)+a \cos \theta=a\left(2 \frac{\sin \theta_{1}-\sin \theta}{\theta_{1}-\theta}-\cos \theta-\cos \theta_{1}\right)=0 .
$$

\section{Points of discontinuity.}

Lemma 1. The intersection of the set of discontinuity points of the mapping $T_{1}$ with $L_{0}=$ $\left[101 \pi / 100, t_{3}\right]$ is finite.

Proof. If $b / a$ is small $t_{2}<101 \pi / 100$. Note that if

$$
\theta^{1}<\theta^{2}, \quad \theta^{1,2} \in L_{0},
$$


then $T_{1}\left(\theta^{1}\right)>T_{1}\left(\theta^{2}\right)$. Indeed, consider the function

$$
h(t, \theta)=b(t-\theta)^{2} / a-\sin t+\cos \theta(t-\theta)+\sin \theta .
$$

The derivative of this function with respect to $\theta$ equals to

$$
-\left(\frac{2 b}{a}+\sin \theta\right)(t-\theta)
$$

and is positive if $\theta \in L_{0}, b / a$ is small and $t>\theta$. If $\theta^{1}$ and $\theta^{2}$ satisfy (12) and $t^{1}$ is such that $h\left(t^{1}, \theta^{1}\right)=0$ then $h\left(t^{1}, \theta^{2}\right)>0$ and the function $h\left(t, \theta^{2}\right)$, negative in a right vicinity of $t=\theta^{2}$ must have a zero on $\left(\theta^{2}, t^{1}\right)$.

So the function $T_{1}$ is monotonous. Note that if $\theta^{1} \in L_{0}$ is a point of discontinuity and $t^{1}=T_{1}\left(\theta^{1}\right)$ then

$$
\frac{d^{2} h}{d t^{2}}\left(t^{1}, \theta^{1}\right)=\frac{2 b}{a}+\sin t^{1}
$$

Note that due to (10) and (11) we have $\sin t^{1}=\sin \theta^{1}+O(b / a)$ which implies that this second derivative must be greater that a fixed negative value. So there exists a $\rho>0$ such that for all discontinuity points corresponding "jumps" of the function $T_{1}$ must be greater than $\rho$. This proves that the number of discontinuity points on $L_{0}$ is finite.

It follows from Eq.(11) that for any discontinuity points $\theta$ of the mapping $T_{1}$ we must have

$$
\cos \theta_{1}=-\cos \theta+O\left(\left(\theta_{1}-\theta\right)^{-1}\right)
$$

Another useful note is that $T_{1}(\theta) \bmod 2 \pi \in\left[t_{2}, t_{3}\right)$ cause we cannot have a transition to a free regime in other points. Meanwhile, for these discontinuity points, the second derivative of solutions (right hand side of Eq. (3)) must be negative, since $x<y$ (delimiter is always to the right of the mass). So, the corresponding value $\theta_{1}$ must belong to $\left[3 \pi / 2, t_{3}\right]$ if $\theta \in\left[t_{2}, 3 \pi / 2\right]$.

In non-degenerate scenarios $((\mathrm{A})-(\mathrm{D}))$ at the moment $t=\theta_{1}$ progression regime starts. The initial velocity of the motion is $x_{1}=-a \cos \theta_{1}+2 b\left(\theta_{1}-\theta\right)+a \cos \theta$. The dynamics of this velocity is described by the formula $\dot{x}(t)=x_{1}+2 c\left(t-\theta_{1}\right)-a \cos t+a \cos \left(\theta_{1}\right)$. The progression regime stops as soon as this derivative becomes negative and the next transition $\theta_{2}=T_{2}(\theta)$ to free flight or to the stop may be found from equations

$$
2 b\left(\theta_{1}-\theta\right)+a \cos \theta+2 c\left(\theta_{2}-\theta_{1}\right)-a \cos \theta_{2}=0 .
$$

Lemma 2. There exists a $b_{0}(q)>0$ such that if $b<b_{0}(q)$ the following statement is true. Starting progression at the point $t_{0}$ with an initial velocity equal to zero, the motion must stop at the instant $t_{4} \in\left[t_{1}, t_{2}\right]$. Consequently, Scenarios $D$ and $D^{\prime}$ are impossible. 
Proof. If $x(t)$ is a solution of Eq.(4) with $\dot{x}\left(t_{0}\right)=0$, we have

$$
\dot{x}\left(t_{2}\right)=-a \cos t_{2}+a \cos t_{0}+2 c\left(t_{2}-t_{0}\right) .
$$

In order to prove that this $\dot{x}(t)$ vanishes somewhere at $\left[t_{0}, t_{2}\right)$ it suffices to prove that the right hand side of Eq. (14) is negative. Instead of this one could prove that

$$
a+a \cos t_{0}-(q-2 b)\left(\pi-t_{0}\right)<0 .
$$

Here we replaced $t_{2}$ with $\pi$ in (14) and respected the fact that $2 c=2 b-q$. If we demonstrate for a fixed $q$ that

$$
a+a \cos t_{0}-q\left(\pi-t_{0}\right)<a\left(1+\cos t_{0}-\sin t_{0}\left(\pi-t_{0}\right)\right)<0 .
$$

then for this fixed $q$ there exists a $b_{0}(q)>0$ such that if $b<b_{0}(q)$ then the estimate (14) is true.

Inequality (15) is equivalent to the following: $\cot \left(t_{0} / 2\right)<\pi-t_{0}$ which is true if $t_{0}>\vartheta_{0}$ (see Eq.(11) ) or, equivalently if $q>\sin \vartheta_{0} a$.

Lemma 3. The map $T$ is such that $T(\theta)=\theta_{2}$ if $\theta_{2} \in\left[t_{2}, t_{3}\right)$. Otherwise, if a motion with stop has been observed (Scenarios $B$ and $C$ ), $T(\theta)=t_{2}$.

Proof. If $\theta_{2} \in\left[t_{2}, t_{3}\right)$ then a motion, starting near the delimiter with the velocity, equal to zero, corresponds to the free regime, so $T=\theta_{2}$. If $\theta_{2} \in\left[t_{3}, t_{0}\right]$, the mass stops until the instant $t_{0}$ then starts moving in progression regime until $t=t_{4}$, stops until $t_{2}$ and proceeds to a free regime. If $\theta_{2} \in\left[t_{1}, t_{2}\right)$ the motion stops until $t=t_{2}$ and also proceeds to the free regime. Since $\theta_{2}$ cannot belong to $\left(t_{0}, t_{1}\right)$, we obtain the statement of lemma.

To finish our proof, we need the following lemma.

Lemma 4. There exist two disjoint subsegments $J_{0}$ and $J_{1}$ of the segment $\left[t_{2}, 3 \pi / 2\right]$ such that $T\left(J_{i}\right) \supset\left[t_{2}, 3 \pi / 2\right]$ and $T$ is continuous on both segments $J_{i}$.

Remark. Then we may say without loss of generality that $T\left(J_{i}\right)=\left[t_{2}, 3 \pi / 2\right]$.

Proof. Let $L_{1}$ be the arc $\left[197 \pi / 100, t_{3}\right]$ and $L_{2}$ be the arc $[101 \pi / 100,51 \pi / 50]$. The first arc is correctly defined if the ratio $b / a$ is sufficiently small.

Let $\theta \in L_{1}$. Consider the solution $x(t)$ such that $x(\theta)=0, \dot{x}(\theta)=0$. Then, direct calculations show that for $b=0$, the considered solution starts from a short period free motion and then stops after a short period of motion with a progression. So, $\dot{x}\left(t_{0}\right)=0$. Due to continuous dependence of the solution on the parameter $b$, the same is true provided the ratio $b / a$ is sufficiently small. In this case, as we have already proved $T(\theta)=t_{2}$. 
Let $z_{1}<z_{2}<\ldots<z_{n}(n \geq 0)$ be discontinuity points of the map $T_{1}$ inside the interval $L_{2}$. Denote $z_{0}=101 \pi / 100, z_{n+1}=51 \pi / 50$.

Suppose that $n<3$. Then there exists $i \in\{0, \ldots, n\}$ such that there is a subsegment $\mathcal{I} \subset\left(z_{i}, z_{i+1}\right)$ of the length not less than $\pi / 500$. Let $\theta \in \mathcal{I}, \theta_{1}=T_{1}(\theta)$.

It follows from Eq. (9) that

$$
\theta_{1}-\theta=\frac{a}{b}\left(-\cos \theta+\frac{\sin \theta_{1}-\sin \theta}{\theta_{1}-\theta}\right)
$$

Consequently, if $b / a$ is sufficiently small, values $\left\{\theta_{1} \bmod 2 \pi: \theta \in \mathcal{I}\right\}$ cover $[0,2 \pi)$. Since $T_{1}$ is continuous on $\mathcal{I}$, we have

$$
\frac{2 b}{a}\left(\theta_{1}-\theta\right) \neq \cos \theta_{1}-\cos \theta
$$

or, equivalently,

$$
2 \frac{\sin \theta_{1}-\sin \theta}{\theta_{1}-\theta}-\left(\cos \theta_{1}+\cos \theta\right) \neq 0
$$

everywhere on $\mathcal{I}$. However, due to Eq. (10), the maximum of the left hand side of inequality (16) is positive while the minimum is negative. So this inequality cannot hold true everywhere.

So, $n \geq 3$. Then it suffices to prove that $T\left(\left(z_{i}, z_{i+1}\right]\right) \supset\left[t_{2}, 3 \pi / 2\right]$ for all $i=1, \ldots, n-1$. Note that estimate (13) implies that $T_{1}\left(z_{i+1}\right)>3 \pi / 2$ if $b / a$ is small. Since $\dot{x}\left(T_{1}\left(z_{i+1}\right)\right)=0$, the corresponding motion proceeds to the free flight immediately after $t=T_{1}\left(z_{i+1}\right)$ and, consequently, $T\left(z_{i+1}\right)=T_{1}\left(z_{i+1}\right) \in L_{1}$.

On the other hand, $T_{1}(\theta) \longrightarrow T_{1}\left(T_{1}\left(z_{i}\right)\right)$ as $\theta \longrightarrow z_{i}+0$ (in the limit case, we have a motion, which "ignores" presence of the delimiter). Since $T_{1}\left(z_{i}\right) \in L_{1}$ the corresponding motion is in the stop regime for $t=t_{0}$ and, consequently, $T(\theta)=t_{2}$ for all $\theta$ from the right vicinity of $z_{i}$. This finishes the proof.

\section{Infinite set of periodic points.}

So, for the mapping $T$ we have obtained two disjoint segments $J_{0}$ and $J_{1}$ which are subsets of the arc $\left[t_{2}, t_{3}\right]$ of the unit sphere such that for both $i=0,1$ mappings $\left.T\right|_{J_{i}}$ are continuous and $T\left(J_{i}\right) \supset J_{0} \cup J_{1}$. Then we prove that for any $m \in \mathbb{N}$ the mapping $T$ has a point of the minimal period $m$. The prove is absolutely standard, but it has to be given, since formally, the existing theorem is applicable to continuous maps of a segment. Also, note that we do not claim that periodic point of a fixed period is unique. There might exist a segment (or, even a Cantor set), consisting of such points. Take a sequence $\left\{\sigma_{k} \in\{0,1\}: k \in \mathbb{N} \bigcup\{0\}\right\}$. First of all, we prove that there exists a point $p \in J_{\sigma_{0}}$ such that $T^{k}(p) \in J_{\sigma_{k}}$ for any $k \in \mathbb{N}$. 
There exists a segment $J_{\sigma_{0} \sigma_{1}} \subset J_{\sigma_{0}}$ such that $T\left(J_{\sigma_{0} \sigma_{1}}\right)=J_{\sigma_{1}}$. Then, we may find a segment $J_{\sigma_{0} \sigma_{1} \sigma_{2}} \subset J_{\sigma_{0} \sigma_{1}}$ such that $T\left(J_{\sigma_{0} \sigma_{1} \sigma_{2}}\right)=J_{\sigma_{2}}$. Repeating this procedure, we obtain a nested sequence of segments

$$
J_{\sigma_{0}} \supset J_{\sigma_{0} \sigma_{1}} \supset J_{\sigma_{0} \sigma_{1} \sigma_{2}} \supset \ldots
$$

The corresponding intersection is non-empty and, consequently contains a desired point $p$.

Fix a number $m$ and consider the sequence $\sigma$, obtained by infinite repetition of a finite sequence $0, \ldots, 0,1$ of the length $m$. Let $I=J_{0 \ldots 01}$ (see above). Then $I \subset T^{m}(I)$ and the mapping $T^{m}$ is continuous on this segment. Applying Weierstrass principle to the continuous function $T^{m}(x)-x$ on the segment I, we obtain a periodic point. Clearly, this point cannot be one of a lower period.

\section{Conclusion.}

Let us formulate the principle result of the paper as a single theorem.

Theorem 1. For all $a$ and $q$, satisfying inequalities (2) there exists $a b_{0}=b_{0}(a, q)>0$ such that for all $b \in\left(0, b_{0}\right)$ the mechanical system, described by equations (3), (44) and (5) is chaotic in the following sense. The phase of transition to free flight of the motion uniquely defines the phase of the next transition. This defines a discontinuous mapping $T$ from the segment $\left[t_{2}, t_{3}\right]$ into itself. Here $t_{2}$ and $t_{3}$ are zeros of the function

$$
F(t)=a \sin t+b
$$

There exist two disjoint segments $J_{0}$ and $J_{1}$ of the segment $\left[t_{2}, 3 \pi / 2\right]$ such that $T\left(J_{i}\right) \supset$ $\left[t_{2}, 3 \pi / 2\right]$ and $T$ is continuous on both segments $J_{i}$. Particularly, there exists an infinite set $P$ of periodic points of the mapping $T$. Minimal periods of points of $P$ are unbounded.

\section{Discussion and future work.}

First of all, let us note that the obtained chaotic dynamics is robust. Of course, we cannot say anything about stability of points of the set $P$. Every particular point of this set may appear or disappear if we slightly change parameters $a, b$ and $q$. The power of the set $P$ may be continuum for some values of parameters, while this set is countable for other values. Neither, the method we offer does not specify the topological structure of the set $P$ and one of its closure.

However, we can select a family of segments $J_{0}$ and $J_{1}$ from the statement of Theorem 1 so that boundary points of these segments locally continuously depend on parameters $a, b$ and $q$. This means that the fact of presence of the one dimensional turbulence in the considered system is robust. The same is true for the fact of existence of an infinite set $P$. Moreover, 
for all fixed values $a, b$ and $q$, satisfying inequalities (2), there exists an $\varepsilon>0$ such that for any $C^{2}$ function

$$
G(t, x, \dot{x}): S^{1} \times \mathbb{R}^{2} \rightarrow \mathbb{R}
$$

such that

$$
|G(t, x, \dot{x})|<\varepsilon, \quad\left|\frac{\partial G}{\partial(t, x, \dot{x})}\right|<\varepsilon
$$

for all $t, x$ and $\dot{x}$ an analog of Theorem 1 is true for the system, where equation (3) is replaced with

$$
\ddot{x}=F(t)+G(t, x, \dot{x}) ; \quad \dot{y}=0,
$$

equation (41) is replaced with

$$
\ddot{x}=F(t)+G(t, x, \dot{x})-q ; \quad \dot{y}=0 .
$$

and equation (15) is the same.

Particularly, the presence of the considered chaotic dynamics must be observed in simulations and experiments. However, in this paper, we are not going to study the general case. We just offer a method how a non-classical chaos may be found. We plan to use this methods for more general systems with a dry friction (see [17] as an example) and provide for these "real life systems" theoretical results accompanied with simulations and experimental data.

Acknowledgements. This second author was supported in part by Russian Foundation for Basic Researches, grant 12-01-00275-a and by Centre for Research and by FEDER funds through COMPETE Operational Programme Factors of Competitiveness (Programa Operacional Factores de Competitividade) and by Portuguese funds through the Center for Research and Development in Mathematics and Applications and the Portuguese Foundation for Science and Technology (FCTFundação para a Ciência e a Tecnologia), within project PEst-C/MAT/UI4106/2011 with COMPETE number FCOMP-01-0124-FEDER-022690.

\section{References}

[1] J. Awrejcevicz, Chaotic motion in a non-linear oscillator with friction, KSME Journal $\mathbf{2 : 2}$ (1988), $104-109$.

[2] M. di Bernardo, Ch.J. Budd, A. R. Champneys, P. Kowalczyk, A. B. Nordmark, G. O. Tost, P. T. Piiroinen. (2008) Bifurcations in Nonsmooth Dynamical Systems, SIAM Review 50:4, $629-701$.

[3] M. di Bernardo, P. Kowalczyk, A. B. Nordmark, Sliding bifurcations: a novel mechanism for a sudden onset of chaos in dry friction oscillators. International Journal of Bifurcation and Chaos, 13 (2003), No. 10, 2935 - 2948. 
[4] B. Blazejczyk-Okolewska, T. Kapitanak, Dynamics of Impact Oscillator with Dry Friction Chaos, Solitons and Fractals, 7:9 (1996), 1455 - 1459.

[5] G. Csernák, G. Stépán, S. W. Shaw. Sub-harmonic resonant solutions of a harmonically excited dry friction oscillator, Nonlinear Dynamics 50:1 (2007), 93 - 109.

[6] B. Feeny, F. C. Moon, Chaos in a Forced Dry-Friction Oscillator: Experiments and Numerical Modelling, Journal of Sound and Vibration 170:3 (1994), 303 - 323.

[7] A. F. Filippov Differential Equations with Discontinuous Righthand Sides, Kluwer Academic Publishers, Dordrecht, 1988.

[8] U. Galvanetto, Unusual Chaotic attractors in nonsmooth dynamic systems, Int. J. Bifurcation Chaos, 15, 4081 (2005).

[9] Gong Cheng, Jean W. Zu Chaotic Motion of a Two-Frequency Forced Oscillator With Dry Friction, ASME 2003 International Mechanical Engineering Congress and Exposition (IMECE2003), November 15 - 21, 2003 , Washington, DC, USA. Proceedings, 641 - 647.

[10] S. J. Hogan, L. Higham, T. C. L. Griffin, Dynamics of a piecewise linear map with a gap, Proc. Royal Society A, 463 (2007), 49 - 65.

[11] A. Katok, B. Hasselblatt, Introduction to the Modern Theory of Dynamical Systems, Cambridge University Press, 1995.

[12] A. M. Krivtsov, M. Wiercigroch, Dry Friction Model of Percussive Drilling, Meccanica 34, $1999,425-434$.

[13] A. M. Krivtsov, M. Wiercigroch, Penetration Rate Prediction for Percussive Drilling via Dry Friction Model, Chaos, Solitons and Fractals, 11 (2000), 2479 - 2485.

[14] P. Kowalczyk, P. T. Piiroinen, Two-parameter sliding bifurcations of periodic solutions in a dry-friction oscillator, Physica D: Nonlinear Phenomena 237:8 (2008), 1053 - 1073.

[15] T. Y. Li, J. A. Yorke, Period three implies chaos Amer. Math. Monthly. 82 (1975), 49 - 68.

[16] O. Makarenkov, J. S. W. Lamb, Dynamics and bifurcations of nonsmooth systems: A survey. Physica D: Nonlinear Phenomena 241:22 (2012), 1826 - 1844.

[17] E. M. Pavlovskaia, M. Wiercigroch, Low-dimensional maps for piecewise smooth oscillators, J. Sound Vib., 305 (2007), 750 - 771.

[18] D. J. W. Simpson, J. D. Meiss Aspects of Bifurcation Theory for Piecewise-Smooth, Continuous Systems, arXiv:1006.4123v1.

[19] A. Stefański, J. Wojewoda, M. Wiercigroch, T. Kapitaniak, Chaos caused by non-reversible dry friction, Chaos, Solitons and Fractals 16, (2003), 661 - 664.

[20] R. Szalai, H. M. Osinga, Invariant polygons in systems with grazing-sliding, Chaos 28(2), 023121 (2008). 
[21] R. Szalai, H. M. Osinga, Arnold tongues arising from a grazing-sliding bifurcation, SIAM Journal on Applied Dynamical Systems 8:4, 1434 - 1461 (2009).

[22] M. Wiercigroch, A. de Kraker, eds. Applied Nonlinear Dynamics and Chaos of Mechanical Systems with Discontinuities. World Scientific: Singapore, New Jersey, London, Hong Kong, 2000 .

[23] J. Wojewoda, T. Kapitanak, R. Barron, J. Brindley Complex Behaviour of a Quasiperiodically Forced Experimental System with Dry Friction, Chaos, Solitons and Fractals 3:1 (1993), 35 46. 